\title{
A retrospective study of clinical, histopathological and direct immunofluorescence spectrum of immunobullous Disorders
}

\author{
Charisma Kunhi Khannan, Dr Ramesh Bhat M \\ Department of Dermatology, Venereology, Leprosy, Father Muller Medical College Hospital
}

\begin{abstract}
Background: Immunobullous disorders are a group of disorders characterized by autoantibodies directed against various skin autoantigens resulting in blister formation. These disorders maybe further classified as intraepidermal and subepidermal depending on the level of bulla. In cases where clinical and histopathological diagnosis could be very challenging, direct immunofluorescence will help at arriving with an accurate diagnosis. Immunofluorescence techniques help in better understanding and classification of the various immunobullous disorders.

Objectives: 1 . The study aims to correlate Direct immunofluorescence (DIF) with clinical and histopathological findings

2. To analyze the utility of immunofluorescence in the diagnosis of these disorders

Materials and methods: The present study included various immunobullous disorders diagnosed by histopathology and immunofluorescence over a period of 2 years from July 2012 to July 2014.

Conclusion: Immunofluorescence helps in the confirmation of diagnosis of immunobullous diseases whenever there is a clinical and histopathological overlap. Individually, none of these methods were conclusive of the immunobullous disorder.
\end{abstract}

Index Terms- Immunobullous disorders, Direct Immunofluorescence, Autoimmune bullous disorders

\section{INTRODUCTION}

$I^{\text {mased }}$ mmunobullous disorders constitute an important group of skin 1 diseases caused by pathogenic autoantibodies directed against target antigens present in the intercellular or dermoepidermal junction.This group may be further subdivided into intraepidermal and subepidermal.Immunofluorescence studies are considered to be the 'gold standard' in the diagnosis of immunobullous disorders and along with the clinicopathological correlation it will help us at arriving with an accurate diagnosis. $^{1,2}$

\section{MATERIALS AND METHODS}

A total of 100 biopsies received over a period of 2 years by the Departments of Dermatology and Pathology were studied ( July 2012 to July 2014 ). Clinical data was collected from the files of department of Dermatology and the same was recorded along with the reports of histopathological and direct immunofluorescence diagnosis. For histopathological examination ( HPE ), skin or mucosal biopsy was obtained from a fully developed vesiculobullous lesion ( lesional biopsy ) and sent in $10 \%$ formalin. Following standard processing, the sections were stained with hematoxylin and eosin and observed under light microscope. For direct immunofluorescence ( DIF ), skin or mucosal biopsy was obtained from perilesional area within $2 \mathrm{~cm}$ diameter of the lesion ( perilesional biopsy ) and was sent in Michel's transport medium immediately where it was stained and seen under immunofluorescence microscope.

\section{RESULTS}

The study group comprised of 50 biopsies. The age group ranged from 4 years (a male diagnosed with EBA ) to 80 years ( a male diagnosed with BP ) with a mean age of 45.52 years. The male to female ratio was $1: 2$. Oral lesions were observed in 4 patients. DIF showed positive findings in 39 skin biopsies, whereas no significant immune complex deposits were seen in 11 biopsies.

In this study, pemphigus vulgaris was the commonest immunobullous dermatosis observed ( 14 ). Of the total, 19 cases ( $38 \%$ ) belonged to pemphigus group, 13 cases ( $26 \%$ ) were bullous pemphigoid ( BP ), 2 ( 4\%) were linear IgA disease ( LAD ), 2 cases ( 4\%) were epidermolysis bullosa ( EBA ), 1 case $(2 \%)$ of dermatitis herpetiformis ( DH ) and 2 cases $4 \%$ were lichen planus pemphigoidus ( LPP ). Out of the 19 cases of pemphigus group, 14 cases ( $68.4 \%$ ) were pemphigus vulgaris ( $\mathrm{PV}$ ) and 5 cases ( 31.5\%) were pemphigus foliaceous ( PF ). 


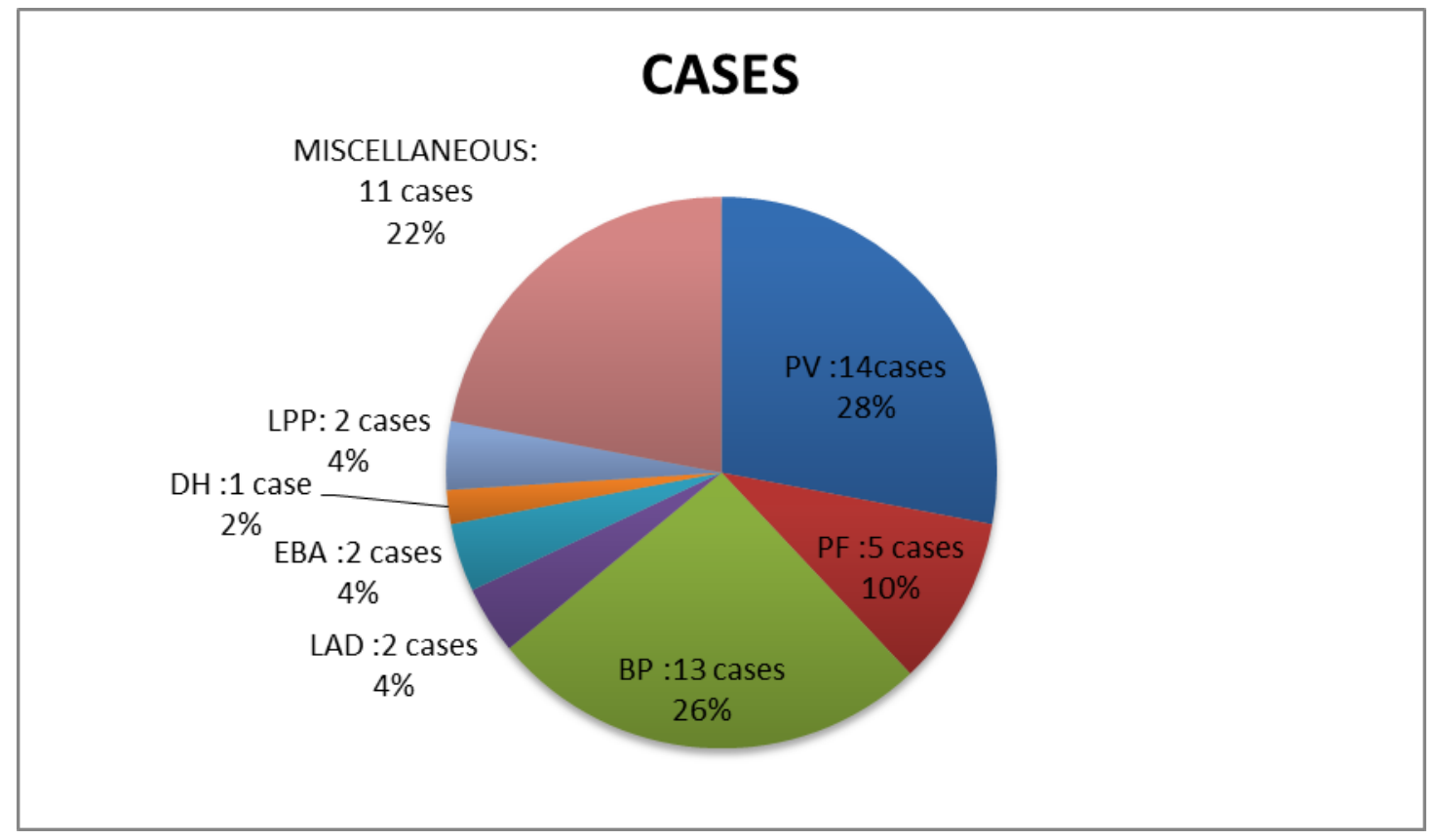

Figure 1: Distribution of immunobullous disorders in this study

Itching and burning sensation of the eroded skin were the common symptoms Vesicles, bullae, erosions and crusting were the most common clinical findings observed. 24 patients of PV, $\mathrm{PF}$, BP had lesions over the trunk ( $48 \%$ ). Involvement of extremities was seen in 26 patients (52\%). Face and scalp involvement was seen in 9 patients (18\%) with PV and PF. Both cases of LPP presented with violaceous papules and plaques apart from vesicles and bullae over normal and involved skin.

Flaccid bullae was the characteristic presentation in 16 ( $84 \%$ ) cases diagnosed with pemphigus. 9 ( $69.2 \%$ ) of the cases diagnosed with BP classically presented with tense bullae over an erythematous base and itching was their most common associated symptom.

On histopathology, all cases of PV showed suprabasal bulla with features of acantholysis. Predominant inflammatory infiltrate consisted of neutrophils.

All cases of PF and BP showed subcorneal bulla and subepidermal bulla respectively. All patients with BP had eosinophils within the subepidermal bulla. LAD, EBA showed subepidermal bulla in all the cases. Both cases of LPP showed subepidermal bulla with basal cell degeneration.

On DIF examination, intercellular space ( ICS ) deposition of IgG was seen in all the cases of pemphigus vulgaris resembling a fishnet pattern. 16 cases out of 19 also showed C3 deposition at the ICS. All cases of PF showed ICS deposition of IgG. All cases of BP showed linear IgG and C3 deposition in the BMZ.
Both the patients of LAD showed linear deposition of IgA along the basement membrane zone ( BMZ ). Subepidermal split was seen in both the cases of EBA. LPP in both the cases showed a linear deposition of IgG and C3 at the BMZ. All except one clinically suspected cases of DH were not confirmed either by histopathology ( HPE ) or direct immunofluorescence ( DIF ). The final diagnosis was based on histopathology and DIF findings.

Table 1: Clinical, histopathological and immunological correlation in $\mathbf{5 0}$ cases

\begin{tabular}{|l|l|l|l|}
\hline DISEASE & $\begin{array}{l}\text { CLINICAL } \\
\text { DIAGNOSIS }\end{array}$ & $\begin{array}{l}\text { CONSISTENT } \\
\text { HPE } \\
\text { DIAGNOSIS }\end{array}$ & $\begin{array}{l}\text { CONSISTENT } \\
\text { DIF } \\
\text { DIAGNOSIS }\end{array}$ \\
\hline PV & 17 & 14 & 14 \\
\hline PF & 6 & 5 & 5 \\
\hline BP & 13 & 13 & 13 \\
\hline LAD & 4 & 2 & 2 \\
\hline EBA & 2 & 2 & 2 \\
\hline DH & 6 & 1 & 1 \\
\hline LPP & 2 & 2 & 2 \\
\hline
\end{tabular}




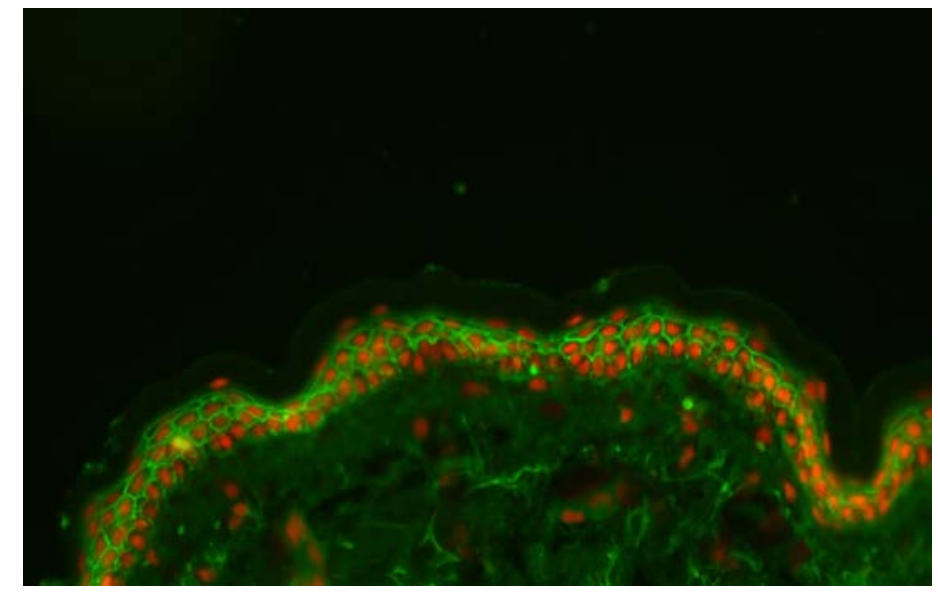

Figure 2: DIF showing pemphigus vulgaris with intercellular deposition of IgG and C3

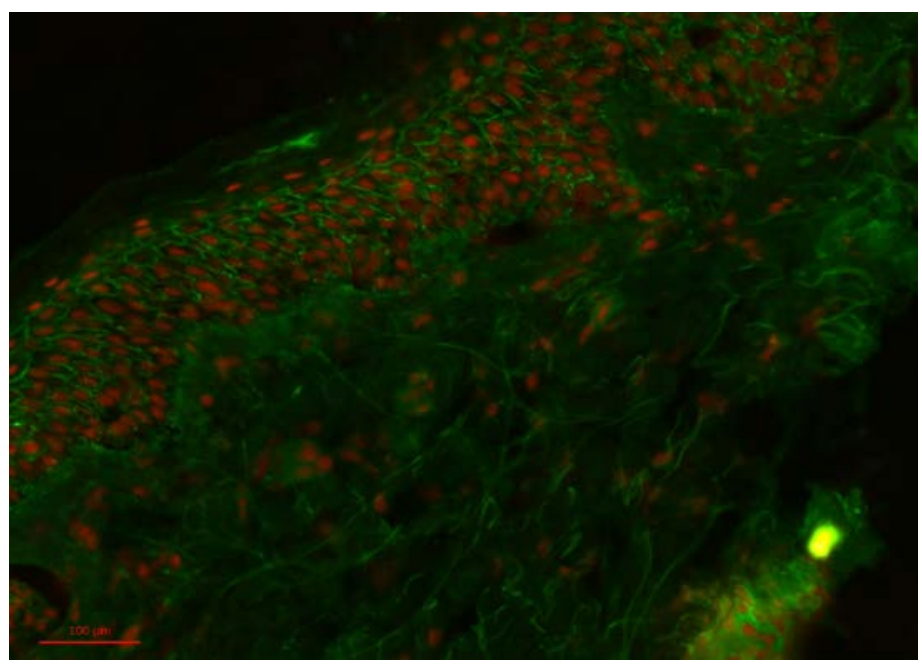

Figure 3: DIF showing pemphigus foliaceous with subcorneal intercellular space deposition og IgG and C3

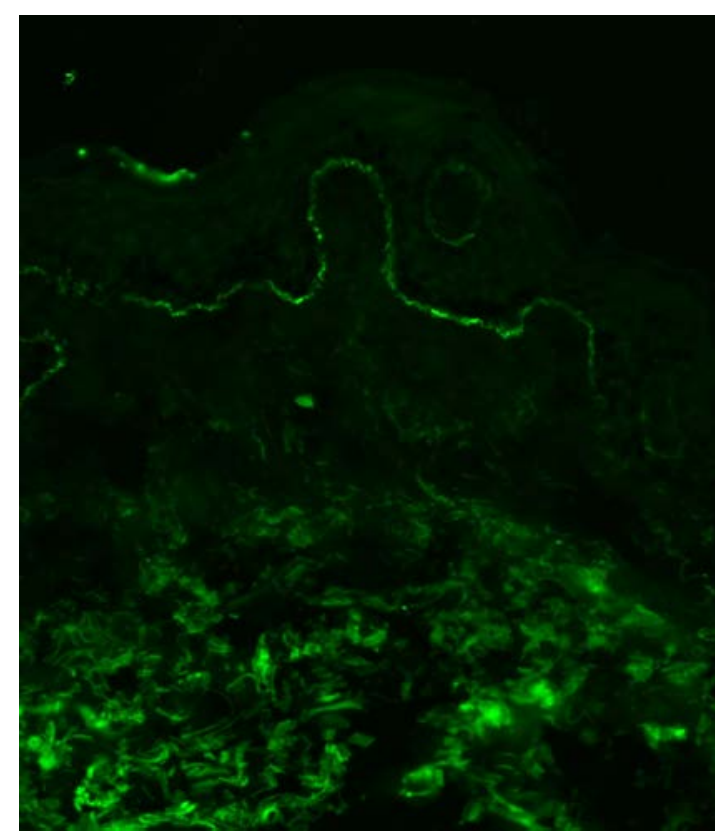

Figure 4: DIF of bullous pemphigoid showing linear deposition of IgG in the basement membrane zone 
Figure 5: DIF showing linear deposits of IgA along dermoepidermal junction

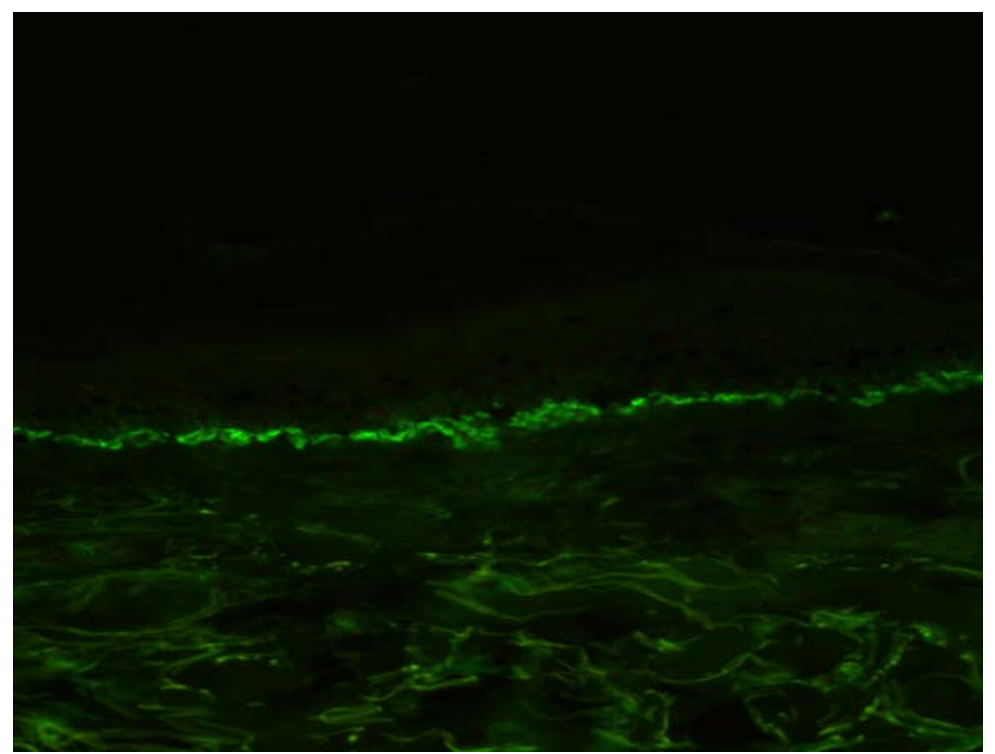

Figure 6: DIF showing LPP with subepidermal bulla subepidermal bulla and IgG, C3 deposits in granular pattern at BMZ

\section{DISCUSSION}

Clinically, all immunobullous disorders may not present with classical morphology and distribution of the lesions. Presentation may vary depending on the severity of the lesions and prior treatment received for the disease. Intraepidermal group of disorders include pemphigus vulgaris and pemphigus foliaceous and subepidermal group of disorders are bullous pemphigoid, linear IgA disease, epidermolysis bullosa acquisita and lichen planus pemphigoidus. ${ }^{1}$

Subepidermal diseases show overlapping histopathology findings and hence it necessitates the use of an additional diagnostic procedure to confirm the diagnosis. DIF demonstrates the immunoglobulin and complement deposited within the skin biopsy specimen and can differentiate the type of immunobullous disorder depending on the type of immune deposits and the pattern of deposition.

Male to female ratio in this study was $1: 2$ which was comparable to other studies done by Shamim et al. ${ }^{3}$ Trunk and extremities were the frequently involved sites in most cases and this was similarly noted in a study done by Shafi et al. ${ }^{4}$ In the current study, Pemphigus ( $38 \%$ ) group of disorders was the most common immunobullous disorder and PV ( $26 \%$ ) was the most common subtype followed by PF (12\% ) and this was in accordance with several other studies. ${ }^{5,6,7,8}$ Oral lesions were evident in 4 patients with PV and was absent in all the cases of PF. Nikolsky sign was positive in 14 ( $73.68 \%$ ) of the cases, in corroboration with other studies. $^{9}$ 
All cases of PV ( $100 \%$ ) showed a suprabasal bulla with acantholytic cells on histopathology. ${ }^{10,11}$

On DIF, all PV ( 100\% ) patients showed IgG deposition in intercellular space and C3 deposition in intercellular space in $84 \%$ of the cases. ${ }^{7,12,13}$

All cases of PF $(100 \%)$ showed a subcorneal bulla on histopathology and DIF showed IgG deposition in the intercellular space. $^{10}$

$\mathrm{BP}$ is most commonly seen in the elderly with the age of onset in their $5^{\text {th }}$ to 7 th decade of life. In the present study, BP occurred in 13 patients. BP classically presented with tense bullae over an erythematous base and itching was their most common associated symptom. It was the most common subepidermal bullous disorder observed in this study. ${ }^{14,15}$ The histopathological findings of a subepidermal bullae with eosinophils in the bulla cavity was also demonstrated by Nishioka K et al. ${ }^{16}$ DIF findings of a linear deposition of IgG and $\mathrm{C} 3$ along the BMZ was consistent with the clinical diagnosis in all cases of BP $(100 \%)$ ). 2 cases clinically diagnosed with pemphigus showed features consistent with BP on histopathology and immunofluorescence.

Out of 4 cases, we arrived at a final diagnosis of LAD in 2 cases $(4 \%)$ showing features of a subepidermal bulla and a neutrophil rich infiltrate with DIF showing linear IgA deposition at BMZ and 1 case showed histopathological and DIF consistent with BP. ${ }^{17,18,19}$

2 cases of EBA were reported of which only 1 showed a positive correlation on histopathology and DIF. In our study, only 1 out of 6 biopsy proven cases of $\mathrm{DH}$ showed immunoglobulin deposits. Both the clinically diagnosed cases of LPP showed positive histopathology and DIF findings.

On analysis, out of 50 cases, 39 ( $78 \%)$ cases of immunobullous disorders showed a positive correlation between clinical, histopathological and DIF findings. In the remaining 11 cases, the clinical diagnosis was different from that of histopathology and DIF pattern. A clinically diagnosed case of pemphigus and a case of LAD, were later confirmed to be $\mathrm{BP}$ with a positive histopathological and DIF correlation. Similar observations such as classical flaccid bullae in pemphigus and tense bullae in BP were described by Khandari et al. ${ }^{20}$

Pemphigus vulgaris with bullae involving only the oral mucosa maybe a sign of early involvement of the disease. In such scenarios, histopathology and DIF helps at arriving at a final diagnosis. ${ }^{11}$

\section{CONCLUSION}

Histopathology and direct immunofluorescence helps in classifying and diagnosing various immunobullous disorders with confusingly similar clinical picture. Individually, none of these methods are conclusive of the immunobullous disorder.

\section{REFERENCES}

[1] Huilgol SC, Bhogal BS, Black MM. Immunofluorescence of the immunobullous disorders part one: Methodology. Indian J Dermatol Venereol Leprol 1995; 61: 187-95.
[2] Vassileva S. Immunofluorescence in dermatology. Int Journal of Dermatol 1993; 32: 153- 61.

[3] Shamim T, Varghese VI, Shameena PM, Sudha S. Pemphigus vulgaris in oral cavity: Clinical analysis of 71 cases. Med Oral Patol Oral Cir Bucal 2008; 13: E622-6.

[4] Shafi M, Khatri ML, Mashina M, Ben-Ghazeil M: Pemphigus A Clinical study of 109 cases from Tripoli, Libya. Indian J Dermatol Venereol Leprol. 1994; 60: 140- 3 .

[5] Huda MM, Afsar MI. A clinicopathological study of pemphigus. Indian J Dermatol 2001; 46: 75- 9.

[6] Zaraa I, Kerkeni N, Ishak F, Zribi H, El Euch D, Mokni M, et al. Spectrum of autoimmune blistering dermatoses in Tunisia: An 11-year study and a review of the literature. Int J Dermatol 2011;50:939-44.

[7] Daneshpazhooh M, Chams-Davatchi C, Payandemehr P, Nassiri S, Valikhani M, Safai-Naraghi Z. Spectrum of autoimmune bullous diseases in Iran: A 10-year review. Int J Dermatol 2012;51:35-41.

[8] Micali G, Musumeci ML, Nasca MR. Epidemiologic analysis and clinical course of 84 consecutive cases of pemphigus in eastern Sicily. Int J Dermatol 1998;37:197-200.

[9] S., Arundhathi, Ragunatha S., and Mahadeva K.C. "A Cross-Sectional Study of Clinical, Histopathological and Direct Immunofluorescence Spectrum of Vesiculobullous Disorders.” Journal of Clinical and Diagnostic Research : JCDR 7.12 (2013): 2788-2792.

[10] Arya SR, Valand AG, Krishna K. A clinico-pathological study of 70 cases of pemphigus. Indian J Dermotol Venereol Leprol. 1999; 65(4): 168-71.

[11] Handa F, Aggarwal RR, Kumar R. A clinical study of 85 cases of pemphigus. Indian J Dermotol Venereol Leprol .1973; 39(3): 106-11.

[12] Kumar S, Thappa DM, Sehgal S. Immunofluorescence study of pemphigus from north India. J Dermatol 1995; 22: 571-5.

[13] Chhabra S, Minz RW, Saikia B. Immunofluorescence in dermatology. Indian J Dermatol Venereol Leprol 2012;78:677-91.

[14] Bernard P, Vaillant L, Labeille B, Bedane C, Arbeille B, Denoeux JP, et al. Incidence and distribution of subepidermal autoimmune bullous skin diseases in three French regions. Bullous Diseases French Study Group. Arch Dermatol 1995;131:48-52.

[15] Wong SN, Chua SH. Spectrum of subepidermal immunobullous disorders seen at the National Skin Centre, Singapore: A 2-year review. Br J Dermatol 2002;147:476-80.

[16] Nishioka K, Hashimoto K, Katayama I, Sarashi C, Kubo T, Sano S. Eosinophilic spongiosis in bullous pemphigoid. Arch Dermotol. 1984; 120: 1166-8.

[17] Egan CA, Zone JJ. Linear IgA bullous dermatosis. Int J Dermatol 1999;38:818-27.

[18] Ingen-Housz-Oro S. Linear IgA bullous dermatosis: A review. Ann Dermatol Venereol 2011;138:214-20.

[19] Rajeev Sharma, Mohammad Nadeem, Mithlesh Chandra: Bullous pemphigoid in childhood, Ind J Dermatol Venereol Leprol, 1996, 62: 100102.

[20] Khandhari KC, Paricha JS. Pemphigus in Northern India Clinical studies in 34 patients. Indian J Dermatol Venereol. 1965; 31: 62-70.

\section{AuTHORS}

First Author - Charisma Kunhi Khannan, MD Postgraduate, Father Muller Medical College Hospital, Mangalore, charismakk@gmail.com

Second Author - Dr Ramesh Bhat M, MD, DVD, DNB, MNAMS, Father Muller Medical College Hospital, Mangalore, rameshderma@gmail.com 
International Journal of Scientific and Research Publications, Volume 5, Issue 9, September 2015 ISSN 2250-3153

www.ijsrp.org 